\title{
Performance of sagnac interferometer with loops made of PCF and PANDA fibers
}

\begin{abstract}
The parametric effects on performance of the Sagnac interferometer with loop (SLI) made of high-birefringence photonic crystal fiber (HB-PCF) and polarizationmaintaining and absorption reducing (PANADA) fibers are simulated and analyzed accordingly in this paper where transmission spectra and the variations of free spectral range are studied under different conditions. The obtained results from calculations and comparisons of the curves loop fiber made of standard polarization-maintaining fiber and high-birefringence photonic crystal fibers revealed that the distance between fringes (DBF) of the SLI would reduce by increasing loop length. This reducing trend in case of HB-PCF has steeper slope. The DBF for two fibers HB-PCF and PANDA with shorter loop lengths would increase.
\end{abstract}

This study reveals that by using high-birefringence PCF, one can optimize the loop length with minimum distance between fringes in the Sagnac loop interferometer. The obtained results provide a required condition for fabrication of SLI-based devices, such as optical sensors, with a small packaging size.

Keywords: sagnac interferometer, loop length, HB-PCF, PANDA fiber, free spectral range, performance
Volume I Issue 6 - 2017

\author{
Faramarz E Seraji,' Fatemeh Pazooki \\ 'Optical communication Group, Iran Telecom Research Center, \\ Iran \\ 2Physics Group, Islamic Azad Univ, North Branch, Iran
}

Correspondence: Faramarz E Seraji, Optical communication Group, Iran Telecom Research Center, Tehran, Iran, Email feseraji@itrc.ac.ir Received: November 20, 2017 | Published: December 13,
2017

and PANDA fibers are simulated and analyzed accordingly, where transmission spectra and the variations of free spectral range (FSR) are studied under different conditions.

\section{SLI with PCF loop}

A schematic diagram of a SLI is shown in Figure 1 in which the loop is made of an HB-PCF [10] or PANDA fiber by connecting two output ports of a directional coupler with a coupling coefficient of $\mathrm{K}=0.5 \cdot{ }^{11}$ The input light to arm 1 of the $3-\mathrm{dB}$ directional coupler is divided into two equal intensity counter-propagating waves, entering the loop from port 3 and 4 . The coupled light to the loop faces a phase delay of $\pi / 2$, thus the intensity of the transmitted light to the arm 2 will equal to the summation of two fields with equal amplitudes, one in clockwise with an optional phase of $\ddot{o}$ and the other field in anti-clockwise with a relative phase of $(0-ð)$. As a consequence, the transmitted field intensity will become zero and the input light maintaining all the energy will reflect back to arm 2 .

The presence of birefringence in the fiber causes a velocity difference and optical path variation related to state of polarization. As a result, when these fields once again enter the directional coupler, will retain a relative phase difference. Therefore, the reflection will become zero and the fields in the coupler recombine, and the resulting light appears at the interferometer output. The interference of the propagated fields in the loop, depending on the birefringence, may either be constructive or destructive.

Irrespective of coupler loss and field evanescence of birefringent fiber of the loop, the transmission $T$ coefficient and reflection coefficient $R$ of the loop are nearly periodic functions expressed as:

$$
T=[1-\cos (\delta)] / 2
$$

In this paper, the parametric effects on performance of the Sagnac interferometer with loop made of high-birefringence-PCF (HB-PCF) 


$$
R=[1+\cos (\delta)] / 2
$$

where $\ddot{a}\left(=2 ð \Delta n_{g} L / \ddot{e}\right)$ denotes the phase difference between polarization modes of high-birefringence where $\Delta n_{g}$ is the fiber group birefringence, $L$ is the loop length, and $\lambda$ represents the wavelength of the light in vacuum.

When the variations of $\Delta n_{g}$ are small in comparison to the wavelength, then $\Delta n_{g} \approx\left|n_{x}-n_{y}\right|$, where $n_{x}$ and $n_{y}$ are the effective refractive indices of polarization modes in $x$ and $y$ direction, respectively. The maximum wavelengths act as resonance wavelengths that are represented as:

$$
\ddot{\mathrm{e}}=\frac{\oslash \Delta n_{g} L}{(2 m+1)}
$$

where $m$ is an integer. The wavelength difference between two adjacent maxima (fringe distance) is determined by the relation

$$
\Delta \lambda=\lambda^{2} /\left(\Delta n_{g} L\right)
$$

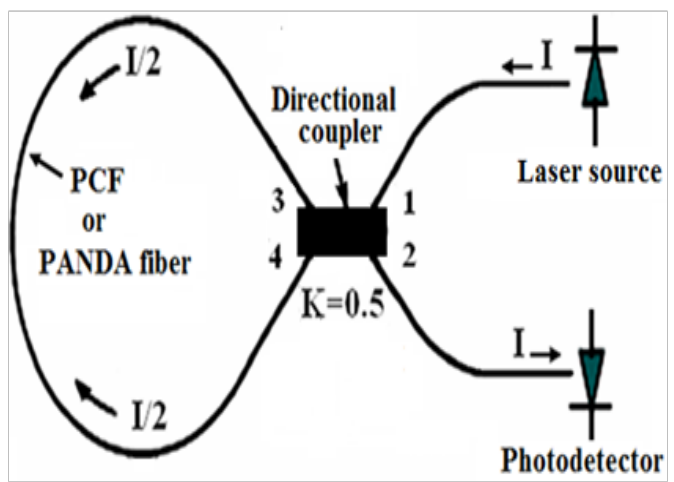

Figure I Schematic of an optical fiber Sagnac loop.

\section{Simulation of transmission spectrum of the SLI}

With reference to Eq. 3, the distance between fringes (DBF), i.e.

$\Delta \ddot{e}$, in SLI is inversely proportional to the loop length. Thus, to obtain the smallest distance between fringes, we need longer loop length made of HB-PCF.

For a comparison, the DBF as a function of loop length of two types of loop fiber, one an HB-PCF with a high group birefringence

of $\Delta n_{g}=8.56 \times 10^{-4}$ and the other a PANDA fiber with group birefringence of $\Delta n_{g}=3.3 \times 10^{-4}$ both at wavelength $\lambda=1550 \mathrm{~nm}$, is depicted in Figure 2.

By comparing the variations of the DBF in terms of loop lengths for the PANDA and the HB-PCF fibers, it is found that the DBF for the two fibers is more for shorter loop lengths. It's further revealed that in HB-PCF, by decreasing the loop length, the trend of increase of the DBF is with smaller slope as compared to the case of PANDA fiber. Further, by reduction of the loop length, the DBF faces more variation when the PANDA fiber is used as the loop.

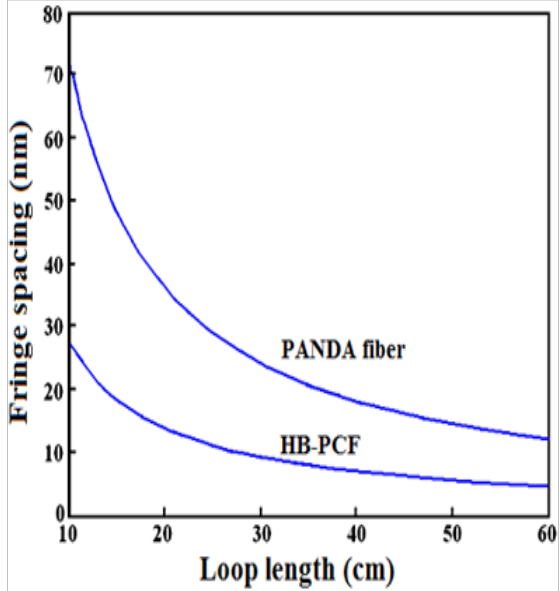

Figure 2 The distance between fringes as a function of loop length for $\mathrm{HB}$ PCF and PANDA fibers.

To study the transmission spectrum and the analysis of spectral response with respect to loop length variations, the simulations are performed by using the solver software MATLAB, as depicted in Figure 3. In Figure 3A, the loop length is taken as $50 \mathrm{~cm}$, whereas in Figure $3 \mathrm{~B}$, the loop is $20 \mathrm{~cm}$ long.
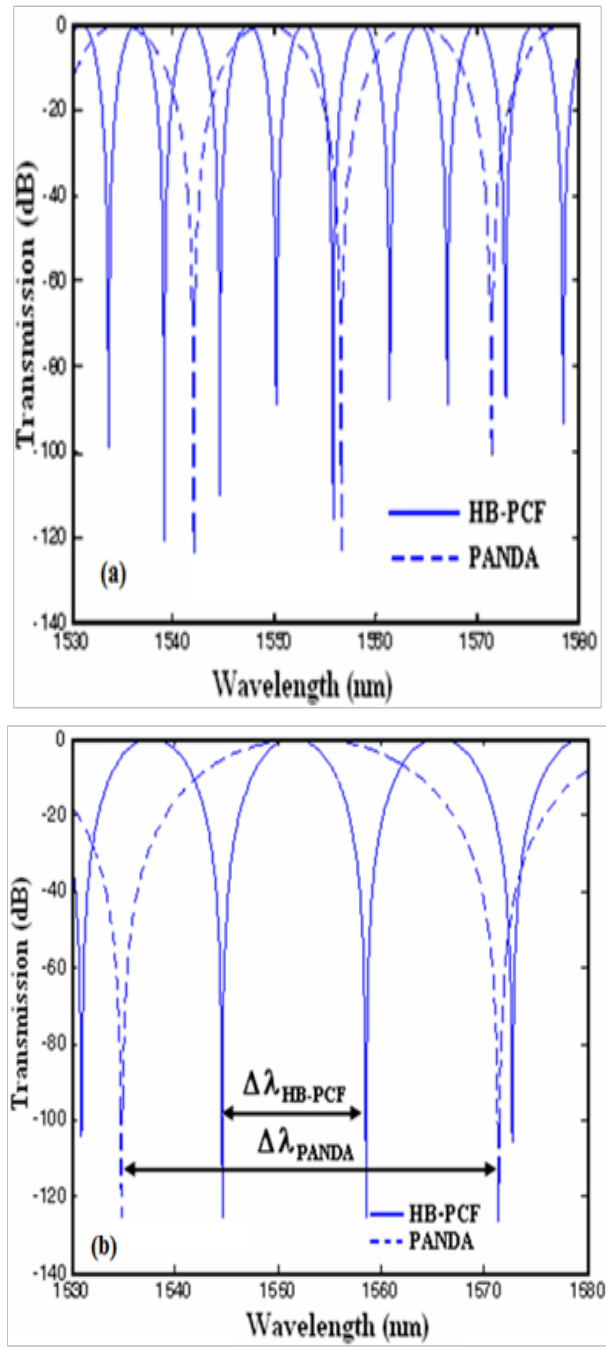

Figure 3 Transmission spectrum of the SLI for HB-PCF loop and PANDA loop with equal loop length of $20 \mathrm{~cm}$. 
On comparing these curves, we note that when loop length becomes shorter, the DBF in SLIs with two types of fiber used in the loop would considerably increase. By assessing Figure 3A \& Figure $3 \mathrm{~B}$, we can extract a relationship between free spectral range (FSR) and group refractive index related to two types of fibers, HB-PCF and PANDA, as follows:

$$
\begin{aligned}
& \Delta \lambda_{P A N D A} \gg 2.5 \Delta \lambda_{H B-P C F} \\
& \Delta n_{g(H B-P C F)} \approx 2.5 \Delta n_{g(P A N D A)}
\end{aligned}
$$

Therefore, by multiplying above expressions, we get:

$$
\left(\Delta \lambda \Delta n_{g}\right)_{P A N D A} \approx\left(\Delta \lambda \Delta n_{g}\right)_{H B-P C F}
$$

If the transmission spectrum of the SLI is illustrated for each fiber for different loop lengths, as shown in Figure 4A \& Figure 4B, the derived relationship between $\Delta \lambda$ and $\Delta n_{g}$ will be similar to the previous expression that is not dependent on the loop length.
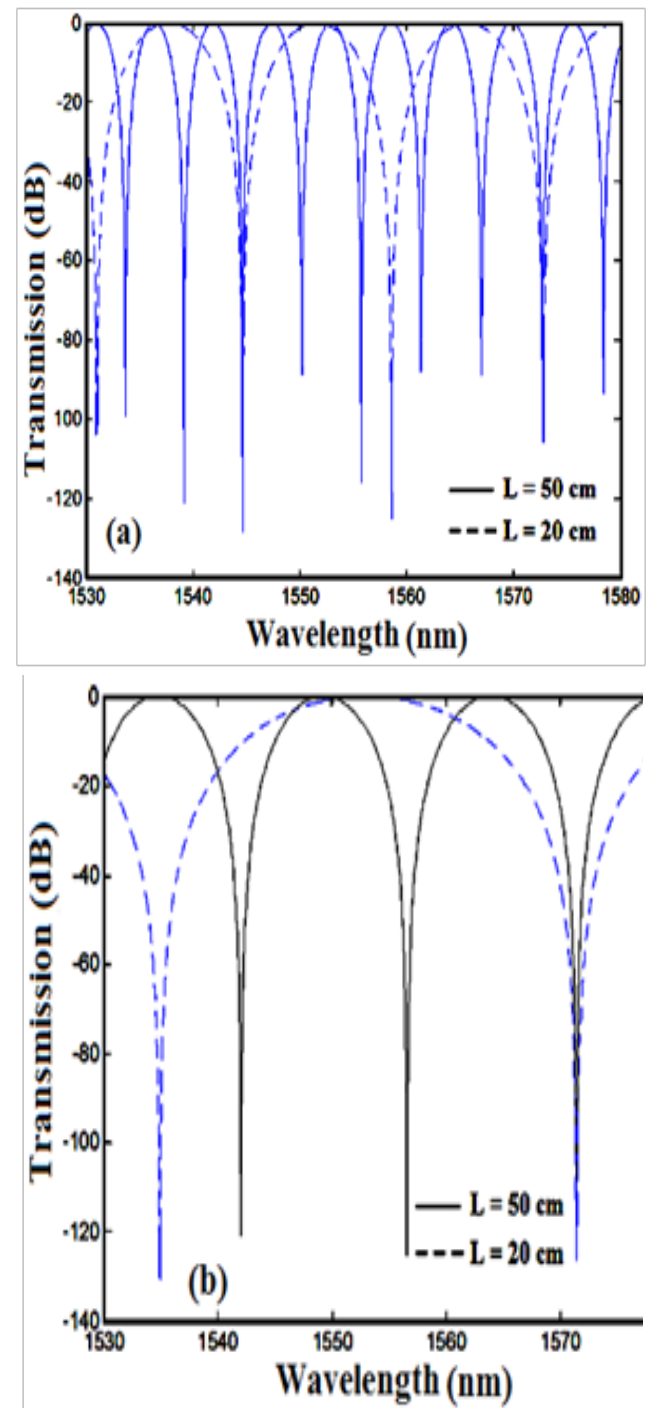

Figure 4 Transmission spectrum of the SLI (A) for HB-PCF and (B) PANDA fiber with different loop lengths.

\section{Variations of FSR in terms of group birefrin- gence}

By using PCF with higher group birefringence, the FSR will reduce. To study the trend of variations, the two expressions considered for two PCFs with birefringence of $\Delta n_{g}=8.65 \times 10^{-4}$ and $\Delta n_{g}=27.67 \times 10^{-4}$, are simulated and then illustrated in Figure 5.

The transmission spectrum of the SLI with a loop length of 20 $\mathrm{cm}$ made of the PCF with birefringence of $\Delta n_{g}=27.67 \times 10^{-4}$ is depicted in Figure 6. On a comparison, one can observe that the DBF of the SLI with a loop length of $20 \mathrm{~cm}$ made of HB-PCF in Fig. 6 is approximately one third of the DBF in Figure 4A.

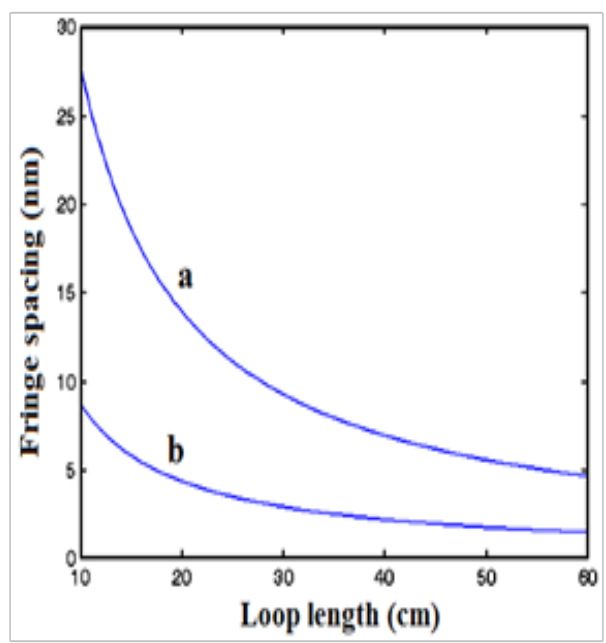

Figure 5 Free spectral range versus loop length made of two PCFs with birefringence (A) $\Delta n_{g}=8.65 \times 10^{-4}$ and (B) $\Delta n_{g}=27.67 \times 10^{-4}$.

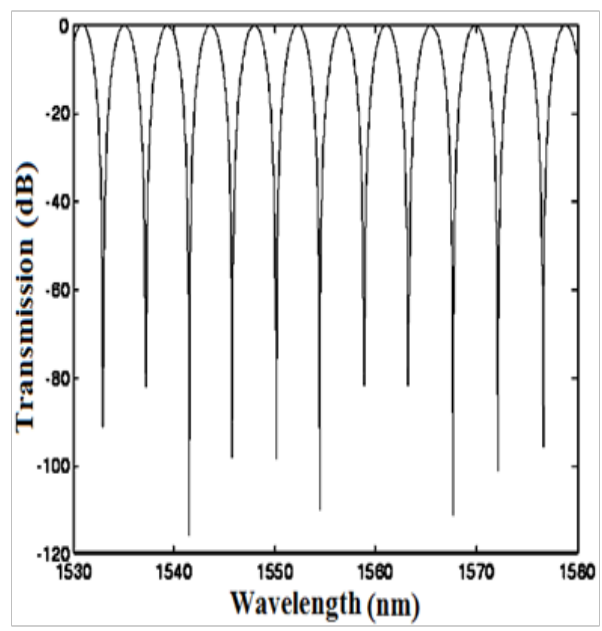

Figure 6 The transmission spectrum of the SLI with loop fiber HB-PCF of $L=20 \mathrm{~cm}$ and $\Delta n_{g}=27.67 \times 10^{-4}$.

\section{Discussion}

The DBF for the loop length of $20 \mathrm{~cm}$ made of PANDA fiber is 36.4 $\mathrm{nm}$, while for the HB-PCF used as the loop fiber, the DBF is obtained as $13.88 \mathrm{~nm}$. The difference of the DBF between two cases is $22.5 \mathrm{~nm}$, or in other words, when miniaturization of SLI is required with short 
loop lengths, employment of HB-PCF is preferred where the value of DBF would reduce more than half of the value.

The comparison between two HB-PCF fibers, one with $\Delta n_{g}=8.65 \times 10^{-4}$ and the other with $\Delta n_{g}=27.67 \times 10^{-4}$ is carried out and the obtained results show that the reducing slope of the curve for HB-PCF loop with group birefringence of more than $\Delta n_{g}=27.67 \times 10^{-4}$ is more and the DBF is less than that of HB-PCF with $\Delta n_{g}=8.65 \times 10^{-4}$.

Yet, in another study, the transmission spectrum of the SLI in terms of wavelength for two fiber samples with loop lengths of $20 \mathrm{~cm}$ and $50 \mathrm{~cm}$ is simulated. The comparison of the distance between maxima in the obtained characteristic curves, certifies the calculated DBF values versus loop length.

\section{Conclusion}

In the present paper, the relationship between the wave length of consecutive maxima in transmission spectrum in terms of loop length of Sagnac loop interferometer is established and analyzed with loop fibers made of HB-PCF and PANDA fiber. The obtained results from calculations and comparisons of the curves loop fiber made of standard polarization-maintaining fiber and high-birefringence photonic crystal fibers revealed that the distance between fringes (DBF) of the SLI would reduce by increasing loop length. This reducing trend in case of HB-PCF has a steeper slope. The DBF for two fibers HB-PCF and PANDA with shorter loop lengths would increase. As an example, the DBF with the loop length of $150 \mathrm{~cm}$ made of PANDA fiber is $\Delta \lambda=14.57 \mathrm{~nm}$, whereas for HB-PCF with equal loop length with $\Delta n_{g}=8.65 \times 10^{-4}$, the DBF is obtained as $\Delta \lambda=5.55 \mathrm{~nm}$. That is, the DBF of SLI with loop length of $50 \mathrm{~cm}$ made of HB-PC, is $9 \mathrm{~nm}$ smaller than the SLI with the loop made of PANDA fiber.

By the obtained results through simulations, this study reveals that by using high-birefringence PCF, one can optimize the loop length with minimum distance between fringes in Sagnac loop interferometer. The obtained results provide a required condition for fabrication of SLI-based devices with a small packaging size.

\section{Acknowledgement}

The authors would like to acknowledge the optical communication group management for permission of publication of the present paper.

\section{Conflict of interest}

The author declares no conflict of interest.

\section{References}

1. Fu HY, Tam HY, Shao LY. Pressure sensor realized with polarization-maintaining photonic crystal fiber-based Sagnac interferometer. Appl Opt. 2008;47(15):2835-2839.

2. Kim G, Cho T, Hwang K. Strain and temperature sensitivities of an elliptical hollow-core photonic bandgap fiber based on Sagnac interferometer. Opt Exp. 2009; 17(4):2481-2486.

3. Mortimore DB. Fiber loop reflectors. J Lightwave Technol. 1988;6(7):1217-1224.

4. Yang S, Li Z, Dong X, et al. Generation of wavelength- switched optical pulse from a fiber ring laser with an F-P semiconductor modulator and a HiBi fiber loop mirror. IEEE Photon Technol. Lett. 2002;14:774-776.

5. Hae Young Choi, Myoung Jin Kim, et al. All-fiber Mach-Zehnder type interferometers formed in photonic crystal fiber. Opt Exp. 2007;15(9):5711-5720.

6. Xiaojun Fang, Richard O Claus. Polarization-independent all-fiber wavelength-division multiplexer based on a Sagnac interferometer. Opt Lett. 1995;20(20):2146-2148.

7. Kim DH, Kang JU. Sagnac loop interferometer based on polarization maintaining photonic crystal fiber with reduced temperature sensitivity. Opt Exp. 2004;12(19):4490-4495.

8. Hansen TP, Broeng J, Libori SEB, et al. Highly birefringent index-guiding photonic crystal fibers, IEEE Photon. Technol Lett. 2001;13(6):588-590.

9. Chun-Liu Zhao, Xiufeng Yang, Chao Lu, et al. Temperature-insensitive interferometer using a highly birefringent photonic crystal fiber loop mirror. IEEE Photon Technol Lett. 2004;16(11):2535-2537.

10. Ortigosa-Blanch A, Knight JC, Wadsworth WJ, et al. Highly birefringent photonic crystal fibers. Opt Lett. 2000;25(18):1325-1327.

11. Wang Y1, Zhao CL, Dong X, et al. A fiber loop mirror temperature sensor demodulation technique using a long-period grating in a photonic crystal fiber and a band-pass filter. Rev Scientific Instru. 2011;82(7):073101. 